$8.8 \%$ and those among general MSM were $17.2 \%, 8.9 \%$ and $4.6 \%$,respectively. Compared with general MSM,MB had significantly higher prevalence of syphilis, HIV, and syphilis-HIV co-infection. In the multivariate logistic regression analysis, MSM aged greater than 30 years, having college or above education level, residing in Shenzhen for 0.5 3.0 years and greater than 3.0 years were less likely to serve as $\mathrm{MB}$ in comparison with those aged less than 30 years, having high school or below education level, residing in Shenzhen for less than 0.5 years. Monthly income more than 5000 Yuan, having drug abuse history, ever being clients of $\mathrm{MB}$, ever reported both insertive and receptive anal sex behaviours will increase the risk of serving as MB among MSM in comparison with monthly income less than 3000 Yuan, never having drug abuse, never being clients of $\mathrm{MB}$ and ever reported predominantly insertive anal sex behaviours.

Conclusion Various factors were associated with MSM serving as $\mathrm{MB}$ and needed to be considered when designing syphilis/ HIV prevention programs targeted them.

\section{P3.97 A SCOPING REVIEW OF PREVALENCE, INCIDENCE AND RISK FACTORS FOR HIV INFECTION AMONGST YOUNG PEOPLE IN BRAZIL}

${ }^{1} \mathrm{P}$ Saffier, ${ }^{1} \mathrm{H}$ Kawa, ${ }^{2,3} \mathrm{G}$ Harling. ${ }^{1}$ Department of Epidemiology, Fluminense Federal University, Brazil; ${ }^{2}$ Department of Global Health and Population, Harvard T.H. Chan School of Public Health, USA; ${ }^{3}$ Research Department of Infection and Population Health, University College London

\subsection{6/sextrans-2017-053264.332}

Introduction Despite young people being a key population for HIV prevention, the HIV epidemic amongst young Brazilians is perceived to be growing. We therefore reviewed all published literature on HIV prevalence and risk factors for HIV infection amongst 10-25 year olds in Brazil.

Methods We searched Embase, LILACS, PsycINFO, PubMed and Web of Science for studies published up to May 2015 and analysed reference lists of relevant studies. We included peer-reviewed studies from any time in the HIV epidemic which provided estimates specific to ages 10-25 (or some subset of this age range) for Brazilians on either: (a) HIV prevalence or incidence; or (b) the association between HIV and socio-demographic or behavioural risk factors.

Results 37 studies in 36 publications met the inclusion criteria: 33 cross-sectional, two case-control, two cohort. Three studies analysed national data. 31 studies provided HIV prevalence estimates, largely for six population subgroups: Counselling and Testing Centre attendees; blood donors; pregnant women; institutional individuals; men-who-have-sex-with-men (MSM) and female sex workers (FSW); two provided HIV incidence estimates. Ten studies showed HIV status to be associated with a wide range of risk factors, including age, sexual and reproductive history, infection history, substance use, geography, marital status, mental health and socioeconomic status.

Conclusion Few published studies have examined HIV amongst young people in Brazil, and those published have been largely cross-sectional and focused on traditional risk groups and the south of the country. Despite these limitations, the literature shows raised HIV prevalence amongst MSM and FSW, as well as amongst those using drugs. Time trends are harder to identify, although rates appear to be falling for pregnant women, possibly reversing an earlier de-masculinization of the epidemic. Improved surveillance of HIV incidence, prevalence and risk factors is a key component of efforts to eliminate HIV in Brazil.

\section{P3.98 SYPHILIS PREVALENCE AND HIV CO-INFECTION AMONGST MEN WHO HAVE SEX WITH MEN (MSM) IN SAO PAULO, 2011 AND 2016}

${ }^{1}$ Igor Prado, ${ }^{1}$ Bruna Robba Lara Redoschi, ${ }^{1}$ Alexandre Welikow, ${ }^{2}$ Erin C Wilson, ${ }^{2}$ Caitlin Turner, ${ }^{2,3}$ Willi McFarland, ${ }^{4}$ Lígia Regina Franco Sansigolo Kerr, ${ }^{5}$ Mark Drew Crossland Guimarães, 'Maria Amélia Veras, The SampaCentro Study Group, Brazilian HIV/ MSM Surveillance Group. 'Faculdade de Ciências Médicas da Santa Casa de São Paulo, São Paulo, Brazil; ' ${ }^{2}$ San Francisco Department of Public Health, San Francisco, USA; ${ }^{3}$ University of California, Epidemiology and Biostatistics, San Francisco, USA; ${ }^{4}$ Universidade Federal do Ceará, Fortaleza, Brazil; ${ }^{5}$ Universidade Federal de Minas Gerais, Brazil

\subsection{6/sextrans-2017-053264.333}

Introduction Syphilis infection rates began an upward trend in the late 1990s, disproportionally affecting men who have sex with men (MSM). Many of these MSM were co-infected with Human Immunodeficiency Virus (HIV). Co-infection often results in a significantly higher burden of disease. Little data examine the prevalence of syphilis in Brazil, the largest country of Southern America. The purpose of this study is to examine disease prevalence and rates of syphilis and HIV co-infection among MSM in São Paulo, the most populous city in Brazil.

Methods This study analyses data from two separate surveys. The first study recruited 771 MSM at randomly selected venues where MSM congregate using time-location sampling (TLS) in 2011. HIV testing was done with all MSM in the field; Syphilis testing was done on a sub-sample of MSM $(n=227)$ who presented to a specialty clinic for screening. The second study recruited MSM by peer referral through respondent-driven sampling (RDS) in 2016. All participants $(n=338)$ were tested for HIV and syphilis. RDS and TLS weights were used in the analysis to provide data representative of the population of interest.

Results In 2011, 19.8\% (CI 13.5-28.1) of MSM were positive for syphilis and $14.7 \%$ (CI 9.2-22.7) were positive for HIV, particularly affecting people within 35-49 years (representing $37 \%$ and $35 \%$ of the syphilis and HIV positive results, respectively). Among the MSM living with HIV, co-infection with syphilis was 45\%. In 2016, 30.67\% (CI 21.8-39.5) of MSM were positive for syphilis and $23.0 \%$ (CI 13.17-32.9) were positive for HIV. In the MSM living with HIV, 48\% tested positive for syphilis. In a Poisson regression, the risk for HIV and Syphilis is higher for 2016.

Conclusion HIV and Syphilis prevalences are at high levels among MSM sampled in São Paulo. An alarming majority of MSM with HIV tested positive for syphilis in 2016. Interventions promoting frequent STI screening among HIV-positive and negative MSM are needed to address both epidemics and mitigate the adverse health outcomes of co-infection and to prevent onward transmission.

\section{P3.99 IDENTIFYING VARIABILITY IN HIV AND SYPHILIS TRANSMISSION RISK IN MSM SEX PARTNER MEETING PLACES}

${ }^{1}$ Jacky Jennings, ${ }^{1}$ Jamie Perin, ${ }^{1}$ Olivia Long, ${ }^{1}$ Khalil Ghanem, ${ }^{1}$ Anne Rompalo, ${ }^{1}$ Errol Fields, ${ }^{1}$ Aruna Chandran, ${ }^{2}$ Shelley Bacchus, ${ }^{2}$ Yvonne Kingdon, ${ }^{2}$ Kehinde Bandemuse, ${ }^{2}$ Patrick Chaulk, ${ }^{1}$ Christina Schumacher. 'Johns Hopkins University, Baltimore, USA; ${ }^{2}$ Baltimore City Health Department, Baltimore, USA

10.1136/sextrans-2017-053264.334 
Introduction Canvassing venues where people meet social or sexual partners is an effective and efficient means of identifying syphilis cases for transmission control and may be important to access MSM at high risk for HIV transmission for PrEP delivery. The objective was to determine variability in the frequency of report and transmission risk across sex partner meeting venues reported by HIV and/or syphilis positive MSM.

Methods Routinely reported public health surveillance data from early syphilis and/or HIV positive MSM between 20092015 in a U.S. mid-Atlantic city were utilised. Past 3 month sex partner meeting venues were collected as a part of routine partner services. Venue geometric mean syphilis titer load (VTL) and HIV viral load (VVL) were calculated and tested using generalised linear additive models with random effects.

Results There were 1870 cases-63\%(1177) syphilis, 37\%(693) HIV. 88\% (1641) were interviewed; among these, 48\% (790) reported $>1$ venue and were on average aged 31(SD10), 78\% (615) Black. Cases reported 1331 venues (avg 2, range 1-16 per case). Syphilis and HIV cases reported 940 (avg 2, range 1-16) and 449 venues (avg 2, range 1-7). Overall and by syphilis and HIV cases, 43\%(577), 45\%(415) and 46\%(208) of reports were for the highest frequency venues $(n=9) ; 3$ internet venues accounted for $66 \%$ of reports. Among the top frequency venues $(n=9$, reports $n=577)$, there was significant $(\mathrm{p}<0.001)$ variability in the report frequency for venues overall (Chi square(CS) 314.7) and by syphilis (CS 225.5) and HIV cases (CS 130). 68\% (642) of syphilis cases had an RPR titer and the VTL was significantly variable (CS 2252.9, $\mathrm{p}<0.001$ ). $35 \%$ (159) of HIV cases had an HIV viral load, and there was not significant variability (CS 5.3, p=0.725).

Conclusion Sex partner meeting venues are similar for syphilis and HIV positive MSM suggesting overlapping transmission networks, although the variability of their report differs. Significant differences venue TL suggest targeting specific venues may be important for transmission control and prevention strategies.

\section{P3.100 HIGH MYCOPLASMA GENITALIUM PREVALENCE IN CHLAMYDIA TRACHOMATIS POSITIVE PATIENTS}

1,2 Dirks JAMC, 'Wolffs PFG, '2Dukers-Muijrers NHTM, 1,2 Hoebe CJPA. Department of Medical Microbiology, School of Public Health and Primary Care (CAPHRI), Maastricht University Medical Centre (MUMC+), Maastricht, The Netherlands

\subsection{6/sextrans-2017-053264.335}

2 Department of Sexual Health, Infectious Diseases, and Environmental Health, South Limburg Public Health Service (GGD), Geleen, The Netherlands

Introduction: Mycoplasma genitalium (MG) is increasingly seen as a clinically relevant sexually transmitted infection (STI), with a clinical spectrum similar to Chlamydia trachomatis (CT) and Neisseria gonorhoeae, including pelvic inflammatory disease and adverse reproductive outcomes. In the Netherlands, MG testing is not currently recommended for first-line STI screening despite a 4\% background prevalence. Very little is known about co-infections with CT or NG as patients are usually only tested after negative CT/NG tests. We therefore studied the co-occurrence of MG and CT in both low- and high-prevalence populations.

Methods 1024 CT-positive participants from the Dutch general population (participants in the Chlamydia Screening
Intervention-study) $(60.3 \% ; 462 \square)$ and STI-clinic in South Limburg, the Netherlands $(39.6 \% ; 259$ \) were retrospectively tested for MG. Men provided urine samples and women selfcollected vaginal swabs. Samples were tested for human cells to ensure adequate sampling. CT/MG co-infections were investigated and correlated to symptoms. Statistical testing was performed using Chi-square test.

Results Of 1024 CT-positive patients, 5.5\% had a co-infection with MG. CT/MG co-infections were present in $6.3 \%$ of the general population, compared to $4.2 \%$ of STI-clinic visitors. $3.9 \%$ of STI-clinic women had a CT/MG co-infection, compared to $7.4 \%$ in the general population. STI-clinic and general population men had a similar MG prevalence of $3.2 \%$ and $4.7 \%$. Symptoms were reported by $37.3 \%$ of patients; $37.2 \%$ in single CT-infections and $39.3 \%$ in CT/MG coinfections.

Conclusion CT/MG co-infections are at least as common $(5.5 \%)$, and in some populations more common (up to $7.4 \%$ ), than in the general (CT-negative) population. As MG-testing is currently not routine practice in most clinics, these women go undiagnosed and receive inferior treatment, which likely contributes to current $30 \%-45 \%$ azithromycin resistance in MG. However, the higher prevalence of MG in the general population and the similar frequency of symptoms in both groups questions the clinical relevance of this pathogen.

\section{P3.101 EFFECTIVENESS OF PRENATAL SCREENING TO PREVENT CONGENITAL SYPHILIS, FLORIDA AND LOUISIANA, 2013-2014}

${ }^{1}$ James Matthias, ${ }^{2}$ Mohammad Rahman, ${ }^{3}$ Daniel Newman, ${ }^{3}$ Tom Peterman. ${ }^{1}$ Centres for Disease Control and Prevention, Tallahassee, USA; ${ }^{2}$ Centres for Disease Control and Prevention, New Orleans, USA; ${ }^{3}$ Centres for Disease Control and Prevention, Atlanta, USA

\subsection{6/sextrans-2017-053264.336}

Introduction Between 2012 and 2014, rates of congenital syphilis increased in Louisiana (LA) (from 52.7 to 73.4 cases per 100000 live births) and Florida (FL) (from 17.4 to 22.1 cases per 100000 live births) ranking them among the highest in the United States. Both states have rules and regulations that require testing pregnant women for syphilis during the first trimester, early third trimester, and at delivery. We evaluated the effectiveness of early and third trimester syphilis screening for the prevention of congenital syphilis in these high-morbidity states.

Methods Reported syphilis cases among pregnant women in FL and LA during January 1, 2013-December 31, 2014, were reviewed for documented screening for syphilis in the first two trimesters and the third trimester. Pregnant women with syphilis were linked to congenital syphilis records and stratified by whether their pregnancy led to a reported congenital syphilis case.

Results 710 pregnant women with syphilis in LA and FL led to 155 congenital syphilis cases. 555 (78\%) potential congenital syphilis cases were averted. 370 (52\%) of the pregnant women with syphilis were staged as early syphilis or high-titer late-latent syphilis, and they were linked to 109 (70\%) of the congenital syphilis cases. 513 pregnant women tested positive for syphilis in the first two trimesters and 470 (92\%) of them had babies without congenital syphilis. Of the remaining 197 women, 109 tested positive for syphilis in the third trimester, and $85(78 \%)$ of them had babies without congenital syphilis. 39 (6\%) women had no reported syphilis screening $\geq 30$ days 